Didier Bottineau

CNRS, UMR 7114 MoDyCo, Université Paris Ouest-Nanterre-La Défense

\title{
Quand le classement est une théorie : le verbe impersonnel dans Les Verbes français
}

\author{
Sous la pluie \\ Il tombe de l'eau, plic! ploc! plac! \\ Il tombe de l'eau plein mon sac. \\ Il pleut, ça mouille, \\ Et pas du vin! \\ Quel temps divin \\ Pour la grenouille! \\ (Jean Richepin)
}

\author{
Il neige il neige sur Liège \\ Et tant tourne la neige entre le ciel et Liège \\ Qu'on ne sait plus s'il neige s'il neige sur Liège \\ Ou si c'est Liège qui neige vers le ciel
}

\section{Des hypothèses aux faits linguistiques}

Ce qui caractérise Les Verbes français (désormais $L V F$ ) par rapport à un dictionnaire ordinaire, c'est le fait que le principe de classification soit très explicitement défini comme la réponse à une question posée par une hypothèse. Celle-ci est formulée dès la première page : «La classification des verbes français repose sur l'hypothèse qu'il y a adéquation entre les schèmes syntaxiques de la langue et l'interprétation sémantique qu'en font les locuteurs de cette langue : à la différence syntaxique entre venir à Paris et venir de Paris correspond une différence sémantique entre la destination et l'origine ». Le verbe, à la différence du substantif, se caractérise prioritairement par le tissu de relations qu'il entretient avec les autres constituants de la phrase.

Diverses représentations et formalisations en ont été formulées, toujours explicitement ou implicitement motivées par une certaine conception de ce réseau et du statut du mot dans l'économie de la phrase en discours et éventuellement de l'unité lexicale en langue.

Tesnière (1959) formalise le verbe comme un noyau (de type atomique) muni d'un potentiel combinatoire (la valence, terme lui-même issu de la chimie: pour former une molécule, des noyaux d'atomes s'unissent par des liaisons de covalence) lié à des satellites, avec le centralisme verbal que cela suppose.

Chomsky postule la pauvreté du stimulus (l'impossibilité d'apprendre une compétence générative en acquisition de la langue maternelle), et sur cette base rend inévitable l'hypothèse d'une grammaire universelle constituant un module syntaxique autonome (phrase $=\mathrm{SN}+\mathrm{SV}$ ), ce qui fait du verbe la tête du SV. Cette approche subordonne le lexique à la syntaxe et permet la sous-catégorisation des verbes par les constructions habituelles auxquelles se prête l'unité lexicale.

Guillaume répond à la même question - par quel type de savoir-faire cognitif un locuteur est-il capable de produire un nombre ouvert de phrases structuralement conformes sans pour autant reproduire des phrases mémorisées ? - en s'adossant au postulat inverse, la richesse du stimulus : le sujet élabore son savoir-produire épilinguistique dans l'expérience du discours, dont l'unité empirique est la phrase. Or le sujet ne mémorise pas les phrases (point commun avec Chomsky), mais les fragments notionnels qui les constituent, les mots du lexique; aussi la compétence syntaxique de production phrastique ne peut-elle enregistrée qu'au niveau des mots par un schème représentationnel spécifiable en discours (le «signifié 
de puissance » qui prévoie notamment le potentiel de réassociation du mot en discours (la «théorie de l'incidence »). Cette approche subordonne la syntaxe au lexique et analyse les structures syntagmatiques et propositionnelles comme le redéploiement d'un savoir-faire combinatoire enregistrées par les parties mises en présence.

Pour sa part, $L V F$ adopte une démarche de compromis. Le schème syntaxique est défini comme le couplage entre les constituants de la phrase et les unités lexicales qui les instancient, et ce schème syntaxique est réputé interprétable. Le dictionnaire consiste donc, pour chaque verbe, à réaliser l'inventaire des schèmes observés dans l'usage et rendre compte de la diversité sémantique à partir des schèmes, ce qui ferait du dictionnaire une lexicographie fondée sur une grammaire de construction avant la lettre (étant entendu qu'un schème syntaxique, très précisément défini, ne coïncide jamais avec les différentes conceptions des constructions qui ont été développées - cf. le $\mathrm{n}^{\mathrm{o}} 26$ des Cahiers du CRISCO). Pour un verbe donné, le nombre d'entrées sémantiques coïncide avec le nombre de schème observés. Ce raffinement engendre la prolifération du nombre des entrées par rapport à ce que l'on observe dans un dictionnaire normal. Pour le premier verbe du dictionnaire électronique, abaisser, le Petit Robert distingue une entrée et deux régimes syntaxiques (transitif, pronominal) ; $L V F, 9$ entrées, dont 6 codées $\mathrm{T}$ (transitif direct) dans la colonne construction et trois $\mathrm{P}$ (pronominal). Cette distribution, contrainte par une démarche sémantique et formelle régulée, coïncide approximativement avec les sous-classes sémantiques observables dans les grands dictionnaires, corroborant le principe selon lequel le schème oriente l'interprétation. Un travail général de comparaison dictionnairique entre $L V F$ et les grands dictionnaires serait à réaliser, mais on peut s'attendre à ce que $L V F$ s'en distingue par le niveau de contrainte imposé dans la distinction des entrées et par la motivation cotextuelle des acceptions sémantiques. Sous cet angle, il fournit un outil de travail intéressant soit pour tester la notion d'invariant sémantico-formel (signifié de puissance en psychomécanique, forme schématique en théorie des opérations prédicatives et énonciatives, invariant métaopérationnel en théorie des phases) soit en spécifiant les conditions de son ajustement contextuel, soit en la réfutant.

\section{Place de l'impersonnel dans $L V F$}

Dans $L V F$, une classe de verbes au moins fait exception : la classe des verbes impersonnels. Dans les dictionnaires, celle-ci est considérée comme allant de soi au point de constituer la première caractéristique signalée pour les verbes essentiellement impersonnels comme falloir (sans distinction de régime de type transitif / intransitif). Dans les grammaires, pourtant, l'impersonnel est considéré tantôt comme un type de verbe, tantôt comme une construction (Charaudeau 1992, 376-377). Dans LVF, falloir fait l'objet de trois entrées, dont deux classées « impersonnel» (X2a pour falloir 01 et 03$)$ et une pas (X1a «auxiliaires, semi-auxiliaires, modaux » pour falloir 02 :

- Falloir 01 / Il f $\sim$ à $\mathrm{P}$ du repos. Il f que $\mathrm{P}$ se repose, constr. N4aA40 (N : verbe transitif indirect, $4:$ sujet « chose », a : complément en $\grave{a}$, donc 4 encode « du repos » comme sujet du verbe ; et A : V. intr., $4: S$ complétif, $0: \varnothing$ circ. pour A,donc 4 encode la complétive comme $\mathrm{S}$ de $\mathrm{V}$. intr.

- Falloir 02 / Il f partir, que P parte. Il f $\sim$ être malade pour refuser, constr. T3500 (T: V. tr. dir., $3: S=$ chose, $5: 0=$ complétive ou infinitif, $0: \emptyset$ complément prép., $0: \varnothing$ circ. ; donc 5 - l'infinitive ou la complétive - encode cette fois l'objet de falloir, et 3 son sujet $i l$.

- Falloir 03 / Il s'en $\mathrm{f} \sim$ de beaucoup que nous puissions faire cela, constr. P4000 (P : pronominal, $4: S=$ complétive ou chose, $000: \varnothing \mathrm{OD}$, cplt. prép. et circ.; la complétive est encodée par le sujet 4. 
Autrement dit, on observe la corrélation suivante : sont reconnues «impersonnelles » les entrées dont le il n'est pas codé $\mathrm{S}$; ne l'est pas celle dont il est codé S. Pour certains verbes lexicaux, on fait le même type d'observation.

La classe $\mathrm{H} 3 \mathrm{~b}$ reçoit les verbes météorologiques, dits impersonnels, que leur sujet soit $i l$ ou le temps. Le verbe pleuvoir fait l'objet de trois entrées :

- pleuvoir 01 / H3b / Il p sur Paris. Il p de grosses gouttes / A41 (intr. + circ.)

- pleuvoir 02 / M3a / Les obus p sur la ville / N3g.

- pleuvoir 03 / E4c / Les nouvelles p au journal en provenance de Chine / N3a.

Seul pleuvoir 01, impersonnel, entre dans la classe H3b (verbes météorologiques) : 02 entre dans M3 « «faire ou imprimer tel mouvement », non-animé » et 03 dans E4 « «(faire) venir de qp ou aller qp », sujet non-animé ». Pleuvoir 01, et avec lui toutes ses variantes suffixées (pleuvasser, pleuviner, pleuvioter, pleuvocher), tous ses synonymes, hyponymes et variantes socio- ou dialectales (flotter, dracher; ou les non signalés doucher et pisser), sont dans cet emploi météorologiques rattachés (ou à rattacher) à la classe $\mathrm{H} 3 \mathrm{~b}$ des impersonnels, avec un schème syntaxique encodé A41 : intr., sujet «complétive ou chose », circonstant obligatoire. $L V F$ ne met pas il pleut en parallèle avec il court (codé A30 : intr., sujet « chose », Ø circ.).

Dans les autres cas, les emplois impersonnels des verbes sont de manière systématique considérés comme un signe de grammaticalisation (domaine GRA, classe X1 ou X2). Ceci concerne certains emploi de verbes comme apparaître (03), s'avérer (02 et 03), arriver (11), et tous les emplois de sembler (01-04), cf. Le soleil paraît / *semble (semble n'a aucun emploi proprement lexical selon ce critère). On constate que $L V F$ distingue deux types d'emploi impersonnel. D'un côté, un impersonnel lexical-sémantique, caractéristique des verbes météorologiques, lié à l'ininterprétabilité du référent du sujet (il pleut) ou son indéfinitude (le temps). L'emploi impersonnel de ces verbes n'est pas considéré comme signe de grammaticalisation : le verbe reste dans sa classe propre (H3b). De l'autre, un impersonnel propositionnel-syntaxique, considéré comme indice de grammaticalisation : pour les entrées qui le caractérisent, le verbe bascule dans la classe $\mathrm{X}$; toutes les entrées sont concernées pour sembler. Remarquons que la classe $\mathrm{X} 2 \mathrm{a}$ des impersonnels pose parfois des difficultés d'analyse en linéarité syntaxique (cf. Sandfeld 1970, 56). Par exemple, demander 13 (il ne demande qu'à pleuvoir) est codé X2a / A40 (intransitif sans circonstant). Si l'on adopte une perspective de type guillaumien (Bottineau 2001) on dira qu'une "proposée de discours » (ici, le couple il / pleuvoir envisagé en amont de tout processus d'actualisation de la relation sujet / prédicat) accède au statut de «transformée de discours » soit par actualisation de cette relation (ce qui donne une connexion sujet - verbe immédiate par le mode indicatif et l'accord personnel : il pleut / pleuvait / a plu etc.), soit par virtualisation de cette même relation (indicatif : il commence à pleuvoir ; subjonctif : il se peut qu'il pleuve). Le subjonctif, forme virtuelle et personnelle du verbe, apparaît lorsque «l'idée regardante » à l'origine de l'interception est munie de son sujet propre (ici, le il impersonnel de $i l$ se peut que), distinct de celui de «l'idée regardée » visée par l'interception (ici, le il impersonnel de il pleuve). L'infinitif, forme virtuelle et impersonnelle ${ }^{1} \mathrm{du}$ verbe, est utilisé lorsque que ce qui devrait être son sujet est détourné par le verbe aspectuel (ou autre) qui en fait son support, étant luimême démuni d'un sujet propre distinct du premier : il pleut, il commence à pleuvoir; je fatigue, je commence à fatiguer. Le verbe aspectuel avec sa préposition s'interpose entre sujet et verbe exactement comme le ferait un auxiliaire, aussi les propriétés du sujet sont-elles celles du verbe infinitif. Tout porte à croire que ne demander qu'à fonctionne exactement de la même manière : il pleut, il ne demande qu'à pleuvoir; elle nous aide, elle ne demande qu'à nous aider - il et elle sont ici les sujets de la proposée de discours il / pleuvoir et elle / nous

\footnotetext{
${ }^{1}$ Dans ces lignes on joue avec plusieurs acceptions de «impersonnel» : on ne confond pas les « sujets et verbes impersonnels » d'un côté et les «modes impersonnels» de tout verbe de l'autre (infinitif et participes, ou le « mode quasi-nominal » de Guillaume).
} 
aider, laquelle est actualisée en transformée de discours disloquée et virtualisée par interpolation d'une idée regardante médiatrice ou intermédiaire en position d'auxiliaire parce que démunie d'un sujet propre, tronquée, structurellement incomplète, ne pouvant former proposition à elle seule (vs il se peut que). Si on accepte cette analyse, il faut alors reconsidérer l'encodage de ne demander qu'à. D'une part, la construction « profonde » de la proposée il / pleuvoir, A41, gagnerait à être explicitée. D'autre part, la construction «de surface », celle du verbe demander à, est celle de demander 03, à savoir N1a (transitif indirect avec préposition $\grave{a}^{2}$. En règle générale, avec cette construction en deux niveaux, on distingue (i) la proposée avec son sujet et sa construction, par ex. celle du verbe météorologique A41 (il + pleuvoir), et (ii) le verbe intermédiaire nécessairement bivalent ( $\mathrm{N}$ : transitif indirect, ou $\mathrm{T}$ : transitif direct). Le verbe interpolé conserve sa propre construction quelle que soit celle de la proposée disjointe, éventuellement impersonnelle : on risque de tout perdre, il risque de pleuvoir. Il existe ainsi une vaste classe de verbes interpolables, perméables à la construction du verbe de la proposée disjoint de son sujet, et qui vont donc admettre un sujet impersonnel en se laissant faire précéder d'un sujet qui n'est en réalité pas le leur. $L V F$ acte ce fait en classant ces emplois X1a, parmi les auxiliaires et semi-auxiliaires grammaticaux, mais n'en tire pas les conséquences pour les emplois de $i l$ impersonnel. Lorsque il est utilisable, c'est que le verbe est véritablement perméable et «laisse passer» un trait inhérent au verbe météorologique sans superposer ses traits propres : il tarde à pleuvoir, ça veut pleuvoir mais ça n’y arrive pas (vs *il échoue à pleuvoir), il continue de pleuvoir (vs *il poursuit de pleuvoir), il a fini / cessé (*terminé) de pleuvoir, etc. Lorsque il est rejeté, c'est que le verbe interpolé contrôle les traits du sujet, le plus souvent le trait animé humain, comme pour les verbes terminer $^{3}$, poursuivre $^{4}$, échouer ${ }^{5}$, ce qui lui interdit à la fois de s'interpoler comme verbe-auxiliaire médiateur et d'admettre un sujet impersonnel commandé par l'infinitif. Pour certains verbes comme risquer, la possibilité de $i l$ impersonnel n'est pas mentionnée dans $L V F$ : cf. risquer 02 / X1a / On r de ne pouvoir rentrer, d'avoir un accident, un accident / T14b0 (transitif) : par commutation on obtient pour la même entrée Il risque de pleuvoir, non signalé. Pour certains verbes comme daigner, cette possibilité est admise implicitement, avec une entrée particulière (5351 / daigner 02 / X1a / T3500) pour la pluie daigne tomber, qui à l'évidence s'articule avec il daigne pleuvoir; ou menacer 05 / X1a / T35b0 la neige menace de tomber, à relier à il menace de neiger. Enfin, pour certains verbes et locutions particulières comme ne demander qu'à, l'emploi avec sujet impersonnel fait l'objet d'une entrée à part. Pour ces emplois particuliers, le trait animé humain ne fait pas obstacle : soit il est accepté dans le cadre d'une espèce d'ironie figée (il daigne pleuvoir), il retrouvant ici sa valeur ancestrale d'entité divine agentive de type «Zeus ${ }^{6}$; soit il est gommé : la neige ne

\footnotetext{
${ }^{2}$ Si tant est que l'on accepte cette analyse : à + infinitif fonctionne-t-il vraiment comme Prép + N ? Je demande à partir ne commute pas avec une nominalisation du type *je demande au départ, structurellement analogue à participer 01 / N1a (participer à la fête). Inversement, risquer 02 (risquer de + INF) est codé T14b0, transitif : risquer de mourir commute avec risquer 01 / risquer sa vie / T13a0, cf. jouer une somme d'argent T13a0, qui serait recodé $\mathrm{T} 13 \mathrm{~g} 0$ pour jouer sa vie.

${ }^{3}$ LVF le prouve : pour terminer, 7 entrées en T1 - transitif direct à sujet animé humain ; et une, en terminer avec, en $\mathrm{N} 1$, transitif indirect à sujet animé humain.

${ }^{4}$ Poursuivre : 7 entrées dont 6 en T1, sauf une, poursuivre 03 / le remords poursuit l'assassin / T3100 : transitif direct, $\mathrm{S}=$ chose, $\mathrm{O}=$ humain), mais $L V F$ ne prévoit pas les emplois métaphoriques où une « chose » comme le remords est vécue et présentée comme une entité anthropomorphisée, ou du moins animée.

5 échouer 05 / A16 on échoue à un examen, dans des négociations. Mais la polysémie de ce verbe rend la situation plus complexe, avec divers emplois transitifs à objet inanimé : le marin échoue le bateau, intransitivable et réversible : le bateau échoue.

${ }^{6}$ En basque il pleut se dit Euria du, littéralement « il (fait de la) pluie, avec un auxiliaire bivalent du à deux actants, l'un absolutif pour euria «la pluie», l'autre ergatif impersonnel pour cet agent occulte. Pour une description des configurations impersonnelles simples et multiples dans la conjugaison agglutinante à accord multiple du verbe basque, cf. Bottineau \& Roulland 2009.
} 
«menace » personne mais se présente analogiquement comme un évènement potentiellement dangereux comme l'est un individu menaçant.

Les problèmes ici soulevés illustrent en quoi une classification résulte d'une théorie, concrètement au nombre de deux : (i) la distinction d'un impersonnel lexical-sémantique et d'un impersonnel propositionnel-syntaxique ; (ii) dans le second cas, la question de savoir s'il faut introduire une classe de verbes grammaticalisés à construction perméable. Tournons-nous à présent vers ce qu'il convient de considérer comme « impersonnel ».

\section{Quelles conceptions de l'impersonnel ?}

L'impersonnel est une catégorie dont le seul nom sonne comme une évidence empirique. Pourtant, derrière cette illusion d'un prêt-à-désigner rassurant, se cache une grande variété d'objets concernés et de conceptions théoriques. Par souci de concision on se contentera d'un tri allusif mais opératoire pour notre propos.

\subsection{Sujet, verbe ou énoncé impersonnel ?}

Le sens du terme impersonnel varie considérablement en fonction des caractéristiques typologiques d'une langue donnée et de la manière dont on l'applique au verbe où à l'énoncé selon des critères sémantiques, morphologiques, et/ou syntaxiques. La notion d'impersonnel sémantique est communément utilisée pour qualifier des verbes pour lequel aucun participant nominal n'est sémantiquement interprétable. Cette définition très largement applicable en typologie se réalise matériellement par des absences de sujet dans les langues sans sujet obligatoire (latin, langues romanes autres que le français) ou par des pronoms à interprétation impersonnelle dans les langues à sujet obligatoire (français : pronom sujet délocutif il ou déictique ça; pronoms sujets neutres it anglais et es allemand); ou, dans une langue comme le basque, par un ou des éléments formateurs agglutinants de $3^{\mathrm{e}}$ personne flexionnelle verbale non reliables à des arguments nominaux spécifiables.

La notion d'impersonnel est appliquée à la morphosémantique grammaticale du verbe pour les formes dépourvues de flexion personnelle (infinitif, participes), l'infinitif étant considéré soit comme asémantique (le «flexif postiche » de Berrendonner 1988), soit comme la marque préparatoire de la catégorie de la personne (la «personne puissancielle » de la psychomécanique). Les verbes à prédication impersonnelle dont le sujet est occulté, voire non rétablissable, ou autre que il / ça, sont exceptionnels : loin s'en faut; peu m'en chaut (on peut y voir à la fois un résidu archaïque de l'époque où ces tournures sans sujet étaient fréquentes, et une remotivation contemporaine par compression du type loin (de là, il) s'en faut (de beaucoup)).

La notion d'impersonnel s'applique également en syntaxe pour les langues à «sujet mobile »: en breton, le sujet se présente soit séparé du verbe sous une forme nominale (clitique ou autre) en position initiale de focale (Me a lenn_ «je li_»), auquel cas le verbe est dépourvu de flexion personnelle; soit sous la forme d'une flexion de personne verbale, si la focale initiale est instantiée par un autre constituant (Bremañ e lennan « maintenant je lis ») ; tout phénomène d'accord est proscrit. Les grammaires, se concentrant sur la morphologie du verbe en tant qu'unité lexicale, parlent respectivement de conjugaisons impersonnelle et personnelle; pour éviter toute confusion entre ce fait de morphosyntaxe propositionnelle indépendant du sens (hormis la focalisation) et l'impersonnel sémantique, j'ai proposé les termes de prédication disjointe et prédication conjointe. Ce contraste concernerait le verbe français pour certaines manifestations locales de la déflexivité (cf. Langages 178) en français parlé actuel (partons / on part). 


\subsection{Inventaire des classes de verbes déclarés «impersonnels » dans LVF}

Par rapport à cette diversité, $L V F$ fait un usage restreint du terme «impersonnel». L'impersonnel sémantique des verbes météorologiques ne fait pas l'objet d'un traitement explicitement singularisé sous cette dénomination. On ne relève que quelques verbes et constructions «impersonnels », c'est-à-dire qui n'admettent que la prédication impersonnelle : falloir 01-03, advenir. Techniquement, un verbe strictement impersonnel est un verbe qui, dans $L V F$, n'apparaît que par une entrée unique dans la classe X2a (43 entrées, p.457) ou H3b (verbes météorologiques).

Pour la première classe, il y en a si peu (9) que l'on peut les énumérer : advenir, apparoir, conster, mésadvenir, mésarriver, mésavenir, refalloir, chaloir, incomber. Seul un lecteur très cultivé connaîtra l'existence de la majorité de ces verbes. Pour un locuteur «normalement cultivé » il en reste trois ou au plus quatre (advenir, refalloir, incomber, et éventuellement chaloir) ; pour un locuteur moins érudit, il n'en reste qu'un, refalloir.

On s'attendrait à trouver dans cette classe X2a le verbe falloir 02 (Il faut partir / Il faut qu'il parte), mais celui-ci est classé $\mathrm{X} 1 \mathrm{a}$ et considéré comme transitif à sujet non-animé (T3500), à la différence de falloir 01 (il faut à $P$ du repos, il faut que $P$ se repose). On comprend que le statut de sujet réel est refusé à l'infinitif et la complétive de falloir 02 : *Partir faut, *Que Pierre parte faut. De ce fait, il est difficile de justifier la différence de classement qui oppose il faut qu'il parte (falloir 02 transitif) à il faut qu'il se repose (falloir 01 intransitif) autrement que par les gloses sémantiques respectives («il convient» pour falloir 01, «il est nécessaire » pour falloir 02), et une construction intermédiaire semble échapper au classement : il lui faut partir, avec cette difficulté que le clitique datif ne remplace pas un nominal lexicalisable (*il faut à $P$ partir). Sans doute l'entrée falloir 02 a-telle été rapprochée par analogie des verbes modaux devoir, vouloir, savoir et vouloir: la classe X1, grammaticale, est définie avant tout par sa fonction et très peu par son sens («aspectuels, modaux »). Du côté de l'actance, l'hypothèse de la transitivité de falloir 02 prête à discussion : en diachronie on retrouve bien ce verbe en association avec un sujet, comme c'est toujours le cas en espagnol actuel avec le verbe faltar (algo falta 'qqch faut'), de construction analogue à celle de manquer (qqch manque / il manque qqch); cf. manquer 08 / Cet article m en magasin / A36, intransitif. Pour ce qui est du verbe manquer lui-même, aucune entrée particulière ne distingue la construction il manque trois élèves, qui ne serait pas analysé comme transitive (vs trois élèves ont manqué le cours : manquer 04 / T1300 A10 ${ }^{7}$ ).

Si on acceptait l'idée d'un falloir 02 intransitif classé X2a en lieu et place de X1a, on se retrouverait, dans $L V F$, avec un unique verbe strictement réservé à la prédication impersonnelle: falloir, avec ses trois entrées et son composé préfixé en re-. Selon cette lecture, à la question: «existe-t-il des verbes exclusivement impersonnels en français d'usage ? » $L V F$ répondrait : «dans le domaine grammatical (classe $\mathrm{X}$ ), en tant qu'unité lexicale, un seul, falloir, en vis-à-vis de locutions recrutant des verbes non intrinsèquement impersonnels, comme avoir dans il y a (pourquoi faut-il toujours ce qu'il n'y a pas ?) ». Autrement dit, hormis falloir, il n'existe pas de verbes strictement impersonnels dans cette classe ; on y trouve uniquement des verbes personnels occasionnellement recrutés par une construction impersonnelle dans certains de leurs emplois, le plus souvent distingués par des tournures particulières (réfléchi : il se peut que ; emploi d'un adverbial dématérialisé : il y a, où $y$ témoigne de la même inconsistance référentielle dans le domaine de l'espace que il dans celui de la personne). Par son organisation, $L V F$ met en lumière le caractèrement extrêmement

\footnotetext{
${ }^{7}$ La construction intransitive A10 correspond à ces élèves ont manqué trois jours. On ne revient pas sur la controverse autour de la relation entre complément temporel non prépositionnel et objet direct. En faveur de l'hypothèse transitive se trouvent les constructions (pseudo-)passives du type les jours manqués ne seront pas rattrapés, dix jours ont été manqués.
} 
volatile de cette catégorie au niveau du lexique français, en net contraste avec la fortune et l'évidence terminologique dont elle bénéficie dans la métalangue grammaticale.

Pour la seconde classe, celle des verbes météorologiques (classe H3b), seuls ceux des verbes hyponymiques dont la morphologie dérivée (pleuviner, pleuvioter, pleuvocher) ne correspond pas directement à une base nominale propre (pluie / pleuvoir, neige / neiger) se comportent comme verbes strictement impersonnels sans «sujet réel» (*il pleuvine des cordes / des obus, il drache des hallebardes), alors que les verbes fréquents l'admettent.

Dans la plupart des cas, $L V F$ adhère à l'idée traditionnelle du sujet réel: tantôt explicitement (tomber 04 : la neige tombe / il tombe de la neige, A36, tous deux intransitifs), tantôt implicitement (entrer 01 on entre dans la maison, $\mathrm{N} 1 \mathrm{j}$ : trans. ind. + dans ; aucune entrée spécifique pour il entre du monde dans le magasin, il faut que j'y retourne). Apparemment, le critère implicite est celui de la fréquence d'usage : il tombe + « sujet réel » est suffisamment courant pour constituer une lexie et être reconnu ou reproduit par les locuteurs comme ce que Lavie (2010, 46-49) analyse comme un exemplaire raccordé analogiquement à un plexus, affectant en retour les propriétés constructionnelles du verbe lexical au point qu'il mérite pour ce type de contexte une entrée spécifique, alors qu'un verbe comme entrer est trop rarement recruté par la construction pour incorporé une configuration schématique-sémantique réalisée par une ligne dans le dictionnaire électronique (de fait, Google ne détecte que peu d'occurrences de il entre du / des »).

\subsection{Le traitement du « sujet réel »}

Derrière le concept mal formulé de «sujet réel » se cache une question tout à fait importante. $L V F$ inventorie pour chaque verbe des schèmes syntaxiques sémantiquement interprétables. Or ce qui apparaît comme un schème syntaxique statique, une fois réalisée la mise à plat par le texte écrit, fonctionne en réalité comme une séquence d'opérations successives lors de la production orale, qu'elle soit destinée à un allocutaire immédiat ou distant (communication adressée par oralisation ou écriture) ou à un allocutaire réflexif (parole intérieure, ou «pensée »: l'endophasie, par laquelle un sujet dirige la matérialisation de sa réflexion à sa propre conscience en recourant aux mêmes ressources lexicales, morphologiques et syntaxiques que s'il conduisait l'avènement du sens à la conscience d'autrui) ${ }^{8}$.

Repensé dans ce cadre dynamique, un schème syntaxique doit être compris comme une procédure de production du sens à partir de la forme (ceci constituant l'inversion parfaite du programme de la sémantique générative, qui génère des formes à partir de représentations sémantiques, et dont découle logiquement la linguistique cognitive). En typologie linguistique, on peut caractériser la syntaxe prototypique de la phrase simple dans une langue donnée en y voyant un modèle de la procédure de d'élaboration du sens (cf. Bottineau 2006 pour une analyse contrastive du français, du basque et du breton).

Or ce que l'on observe, c'est que le sujet n'est pas nécessairement exprimé par un constituant unique qui recevrait le couplage matière (notionnelle) / forme (grammaticale) en une seule étape. Au contraire, on constate souvent que le sujet se construit progressivement. En breton, on trouve fréquemment des énoncés du type Pêr a zo brav e ti 'Pierre est beau sa maison' "Pierre a une belle maison », avec un sujet transitoire Pêr figurant un ensemble, le

\footnotetext{
${ }^{8}$ Il faut distinguer la pensée consciemment contrôlée et que viennent refléter des formes verbales des processus de pensée incontrôlés, portés par des actes de langage non maîtrisés mais qui portent leur propre contenu à la conscience. C'est toute la différence entre je pense / crois que d'un côté et il me semble de l'autre, ou en basque gustatzen dut «j'aime ça » (avec un ergatif et un absolutif) vs gustatzen zait «ça me plaît » (avec un datif et un absolutif). Maillard (2010, p.112) formule très explicitement cette hypothèse du statut psychologique des constructions impersonnelles : «Ce qui est en jeu avec les constructions impersonnelles, ce n'est rien moins que le véritable statut logique et psychologique de la pensée. »
} 
possesseur pris pour repère, et un sujet terminal $e$ ti, qui sélectione définitivement une unité au sein de l'ensemble pris pour repère (l'allocutaire est informé par avance par la prosodie que le sujet initial est transitoire) : le sujet «apparent » est un sujet transitoire, et le «sujet réel » le sujet terminal une fois réalisée la correction spécificatrice. En français, on observe de tels découplages entre la matière et la forme :

(i) la matière est donnée avant la forme : mon père, il est parti. Le «sujet» est construit d'abord notionnellement, puis reçoit sa fonction par le pronom, ce qui permet de conduire une opération d'introduction ou de présentation à l'adresse de l'allocutaire en vue d'amorcer la prédication par un sujet effectivement disponible à l'esprit. Il est ressenti comme personnel parce que la préséance de la matière sur la forme permet la récupération anaphorique du référent.

(ii) la matière est donnée après la forme : il est arrivé un accident. Le « sujet » est construit d'abord comme support formel de la prédication par le pronom («sujet apparent », décrété tel parce qu'il commande l'accord), et ensuite alimenté notionnellement par le «sujet réel », la «séquence impersonnelle » de la Grammaire méthodique du français (8.3.1), le « complément » ou « complétif du sujet » de Le Bidois \& Le Bidois (1935, §319, 177-178; pour la complétive, 1938, §1271, 323) avec sa valeur «d'explicitation » - implicitement, pour l'allocutaire - d'un «sujet vague et indéterminé»; le «prétendu sujet logique des impersonnels » (Brunot 1936, 289-291).

L'effet sémantique est connu: le sujet réel est rhématique et entre dans la zone syntaxique des «informations nouvelles », ce qui permet de lui assigner une fonction de réplique dans le cadre d'un enchaînement syntaxique à caractère simulativement dialogal du type il est arrivé (qqch - quoi ?) un accident: la rhématisation force une interrogation anticipée chez l'allocutaire, à laquelle vient opportunément répondre le sujet notionnel différé. Pour que la chose fonctionne, il faut en principe que le verbe ne sélectionne pas de lui-même l'identité du sujet : on imagine assez mal il aboyait un chien, aboyer ne soulevant guère de question à laquelle chien soit susceptible de répondre. Mais dans une énumération comme : Dans l'Arche de Noë, il aboyait des chiens, il miaulait des chats, il piaillait des oiseaux, il sifflait des serpent, il rugissait des lions, il feulait des tigres, il hurlait des singes, il hululait des chouettes, il caquetait des poules, il coassait des grenouilles, il bêlait des moutons, il meuglait des vaches, il brayait des ânes, il blatérait des chameaux... la stratégie de présentation des animaux à partir du cri symptomatique rend cette construction a priori imprévisible tout à fait recevable. Il y avait un chien qui aboyait est plus complexe et permet une double rhématisation, du sujet d'abord, du prédicat ensuite, en passant par une délégation énonciative par la relative. L'effet de sens est l'anomalie de l'action constatée (qui aboyait au lieu de dormir, éventuellement au lieu de dormir comme les autres; avait-il repéré un intrus?).

Rousseau (2010, 50-51) insiste sur la nécessité structurale de $i l$ dans ces constructions et son rôle dans l'économie générale de l'énoncé : la linéarité manifeste une transition d'un pôle énonciatif initial centré sur le locuteur (avec notamment les opérations de modalisation) à un pôle référentiel terminal, et des hiérarchies analogues ont pu être observées par ailleurs pour l'ordre des adjectifs dans le syntagme nominal anglais et l'ordre des auxiliaires dans le syntagmes verbal des langues germaniques, et De Carvalho (2010, 61-63) signale que la forme impersonnelle est implicitement révélatrice de la subjectivité du moi dans l'acte de présentation du sens. Si $i l$ est «référentiellement vide», il se charge d'autant plus d'une valeur métalinguistique de prise en charge élocutive, de modalisation et de gestion grammaticalement explicite du rapport allocutif.

De tels mécanismes sont décrits pour le français parlé par Morel (2010) par le concept de décondensation et engendrent des phénomènes de déflexivité, mais à la limite on peut se demander si l'état décondensé n'est pas la règle et la condensation, l'exception (type le chien 
aboie, la caravane passe). Pour le verbe rester de il reste du vin dans la bouteille / du pain sur la planche, il est rare de trouver des emplois non impersonnels correspondant à $L V F$ rester 08 «subsister» / Rien ne r aujourd'hui de sa fortune. I A35 : ? du vin reste dans la bouteille (partitif incompatible avec le rôle de sujet thématique, au même titre que *un chat est sur le toit) ; l'exemple de $L V F$, avec le quantifieur négatif (rien) extrait du partitif (rien de sa fortune), crée les conditions de la thématisation avec l'effet de présupposition. Il en va de même pour il reste du pain sur la planche (valeur présentative: le locuteur amène l'allocutaire à prendre conscience du problème) que l'on rencontre parfois sous la forme non impersonnelle du pain reste sur la planche (ici le locuteur part du principe que l'allocutaire est conscient du problème, partage son point de vue $)^{9}$. Sandfeld (1970) signale une particularité $\mathrm{du}$ « pronom » en $: d u$ vin reste dans la bouteille / *en reste dans la bouteille vs il reste $d u$ vin dans la bouteille $\rightarrow$ il en reste dans la bouteille. Le pronom il remplace un nominal défini (l'homme / il vient), les pronoms indéfinis on et quelqu'un conviennent pour un nominal indéfini, mais l'emploi de en dans un rôle clitique n'en fait pas pour autant un pronom admissible en fonction de sujet. Pour tous ces exemples, le plus simple est de considérer que rester 08 requiert un sujet disjoint de la forme sujet apparent + sujet réel, lequel ne peut être conjoint en «sujet tout court» que si certaines conditions sont satisfaites en matière de thématicité : on ne peut pas considérer la construction il reste qch comme une variante impersonnelle de qch reste, c'est l'inverse qui se produit (une «intraposition » en lieu et place de l'extraposition de la grammaire générative). Autrement dit, rester 08 gagnerait à recevoir une spécification supplémentaire. Son synonyme subsister ne pose pas le même problème.

Les propriétés bien connues du sujet réel s'accordent de cette analyse: le sujet terminal tend à être étendu, à être indéfini ou négatif (ou défini avec comparaison : il m'arrive la même chose qu'à toi), à recevoir des spécifications quantitatives (il arrive de plus en plus de monde $)^{10}$, à manifester une démarche de comptage et de reconnaissance graduelle qui inscrit la prise de conscience interprétative de son identité dans la durée psychologique de l'heuristique, elle-même indexée sur celle de la progression discursive linéaire. Dès lors que l'on conçoit les marqueurs des sujets apparent et réel comme les étapes disjointe de la construction d'un sujet disloqué pouvant être parcourues dans un sens ou dans l'autre, la notion de «sujet réel » perd de son approximation et peut être validée, éliminant du même coup la nécessité de voir de la transitivité là où il n'y en a pas, mais permettant aussi de comprendre pourquoi on ressent à l'interprétation un air de famille entre l'objet et le sujet réel (qui de fait s'excluent mutuellement, comme s'ils relevaient du même paradigme distributionnel) : l'un et l'autre répondent à la question soulevée par le verbe (il arrive quoi ? - du monde ; j'ai mangé - quoi ? - tout le chocolat). Les structures Sapp-V-Sréel et SVO instancient, chacune à sa manière, le même schème syntaxique de mise en scène pseudodialogale [entité 1 - processus - quoi ? - entité 2], les entités 1 et 2 étant toutes deux notionnellement spécifiées dans le cas de SVO, alors que dans Sapp-V-Sréel Sapp est sémantiquement vide et se contente de saturer la position syntaxique dans le seul but de repousser Sréel en position finale où se lui voit conférer le statut pseudo-dialogal de réplique à une question implicitement prêtée à l'allocutaire (réel ou figuré). On estime donc que pour dans $L V F$, (i) divers cas de transitivité discutable peuvent être résolus en consolidant la notion de sujet réel, et (ii) les divergences de traitements entre des verbes comme entrer vs arriver s'expliquent par la reconnaissance que le fait d'usage de la décondensation se fige ou non au niveau du lexème verbal selon la fréquence de ses emploi, et la probabilité pour cette

\footnotetext{
${ }^{9}$ Cette différence correspond aux configurations 1 associative et 2 dissociative de l'interlocution selon Douay et Roulland dans le cadre de la théorie des relations interlocutives, cf. Douay 2000.

${ }^{10}$ Grevisse 1936 donne cet exemple du Médecin de campagne de Balzac: Tous les lundis, il part maintenant pour Grenoble plus de soixante charrettes.
} 
coalescence s'opère est relativement prévisible (cf. supra le cas de aboyer). Ceci semble conforme à l'esprit de la notion de schème syntaxique et de participation des relations cotextuelles au profilage des propriétés lexicales, à la fois sémantiques et formelles.

\subsection{La signification de $i l$ et ça}

La question de savoir si il impersonnel est pourvu ou non d'un sens a été abondamment discutée. Guillaume et à sa suite Moignet y ont vu une «personne d'univers » : la désignation d'une représentation globalisée de l'ensemble de l'environnement dans lequel s'inscrit la relation je / tu («le petit face-à-face », dont la formalisation est à la base de la théorie des relations interlocutives de Douay et Roulland). La meilleure méthode pour répondre à cette question consiste sans doute à appliquer (avec circonspection) le test de la commutation il / $c ̧ a$ : il est «sémantique » lorsqu'il est opposable, et il ne l'est pas lorsqu'il ne sert qu'à différer le sujet réel (décondensation). Dans $L V F$, la question de ça est évoquée pour les verbes météorologiques, mais le test n'est pas systématique. En français «standard», il impersonnel s'oppose au couple je/tu (interlocution = locuteur/allocutaire) par la délocution et relègue la personne d'univers à l'extérieur du couple interlocutif, alors que ça, déictique, réalise son inclusion à la sphère de l'expérience immédiate conjointe à celle de la parole. Il pleut est un constat plutôt intellectuel, qui ne met pas l'accent sur la manière dont l'évènement concerne ses observateurs perceptuellement et psychologiquement ; ça pleut fait de la pluie un acteur de l'expérience immédiate.

Dans les variétés du français qui opposent $i l$ et ça, le second s'accommode des contextes émotionnels expressifs spontané peu regardants de la bienséante réserve des gens convenables : qu'est-ce que ça pleut / drache / pisse / jute, dis-donc! Mais dans diverses variétés régionales du français parlé populaire (savoyard ${ }^{11}$, franco-provençal, bourbonnais ${ }^{12}$ ) l'emploi de ça est la règle, peut-être en raison même de la portée pragmatique de la remarque météorologique. Dans $L V F$, ça s'accommode difficilement des verbes que l'on perçoit comme littéraires, mais parfaitement avec ceux que l'on identifie à des usages décontractés et/ou régionaux: l'hétérogénéité sociolinguistique des entrées rend l'effet sémantique parfois difficile à apprécier (et la question n'est pas anodine dans un dictionnaire qui, justement, indexe l'interprétation sur le schème syntaxique). Le problème se pose différemment dans la classe X2a : ces verbes n'acceptent généralement que il de décondensation (il faut que / *ça faut que), sauf ceux qui admettent une référence anaphorique - il se peut qu'il vienne vs ça se peut, où ça déictique pointe la réplique antérieure érigée en objet discursif et la constitue en thème promis à un commentaire, le jugement de probabilité. L'opposition il / ça concerne aussi bien les verbes sémantiquement impersonnels (verbes météorologiques) que les verbes syntaxiquement impersonnels (à décondensation), mais selon des articulations sémantiques distinctes : exclusion / inclusion à la sphère interlocutive d'ordre référenciel pour le premier et discursif pour le second. En outre, certains usages parlés et régionaux tendent à utiliser ça comme opérateur de décondensation progressive (cataphorique) : ça se peut qu'il vienne, où ça peut soit pointer un propos déjà évoqué (anaphore discursive : reprise « horizontale » d'un déjà dit dans l'avant-texte), soit pointer un propos déjà envisagé par le locuteur mais encore non mentionné explicitement (anaphore génétique dans une perspective guillaumienne: reprise «verticale » ou «en profondeur»d'un déjà pensé ou conçu sur l'axe du temps

\footnotetext{
${ }^{11}$ Cf. également ce de c'est quatre heure, signalé comme «provincial (spécialement suisse) par Wartburg \& Zumthor 1947, §665, 182. En interrogation, ce tour dénote une réaction émotionnelle expressive à une situation de prise de conscience du temps qui s'écoule.

${ }^{12}$ Cf. la définition de temps vert dans le Dictionnaire bourbonnais, le parler de Diou : type de temps caractérisé par un ciel entièrement voilé, un vent faible ou nul, une température fraîche. Ça fait trois jours qu'on a ce vieux temps vert, ça sait pas si ça veut pleuvoir ou pas, mais ça fait pas chaud pour la saison. http://projetbabel.org/diou/index.htm
} 
opératif, une proposée de discours). On ne peut donc pas faire du test $i l$ / ça un test probant pour distinguer les impersonnels sémantiques et structuraux.

\section{Verbes météorologiques}

Les verbes météorologiques font dans $L V F$ l'objet d'un traitement particulier. Deux analyses sont possibles, l'une sémantique en termes de représentation cognitive (image schema), l'autre structurale en termes de prédication.

Du point de vue de la grammaire cognitive, qui a diversement formalisé la chose, un évènement comme la pluie est représentable par une image schématique impliquant une source (le nuage, ou Zeus), un trajecteur (les gouttes), une trajectoire (l'axe de la chute, négocié entre la force de gravité et le vent), une cible (le sol, le lieu géographique d'impact). Pour un modèle qui formalise le schème syntaxique en tant que vecteur de l'interprétation, il est utile de s'interroger sur la relation liant le choix métalinguistique d'encodage et la configuration de la représentation sémantique, cognitive ou non, à laquelle elle pourrait correspondre (la grammaire cognitive fournissant alors des modèles topologiques des configurations représentées. On n'est pas obligé pour cela de considérer que les structures encodent des représentations mentales, mais plutôt qu'elles en vectorisent la production (au sens théâtral du terme $)^{13}$.

En basque, il pleut se dit Euria du 'pluie-la la-(fait)-il' : l'auxiliaire agglutinant reçoit deux indices actanciels, l'un absolutif correspondant à euria "la pluie », l'autre à la source, l'agent fantôme formellement marqué mais non explicitable lexicalement : dans ce verbe c'est le constituant ergatif qui est impersonnel ${ }^{14}$, et la structure se glose «il (a / fait) la pluie » où $i l$ est impersonnel mais non moins agentif, causateur ou source. L'image schématique combine deux entités, une source (l'ergatif impersonnel) et un trajecteur (euria); pas de mode de déplacement, de trajectoire ni de cible.

On pourrait envisager une telle analyse pour le français il tombe de la pluie/neige / l'eau / des cordes / des hallebardes, il pleut des cordes / des hallebardes, ou l'anglais it's raining cats and dogs (vs *cats and dogs are raining), avec une source il, une trajectoire tombe, un trajecteur percevable pluie/neige ou métaphoriquement imaginable cordes/hallebardes, et la fusion du trajecteur et de la trajectoire dans le même verbe lexical dans les cas d'incorporation nominale ( «chute de pluie »= pleuvoir, «chute de neige »= neiger), ce qui entraînerait l'introduction d'entrées munies d'un schème syntaxique de type $\mathrm{T}$; tel n'est pas le choix de $L V F$.

En revanche, dans $L V F$, pour tous les verbes de type $\mathrm{H} 3 \mathrm{~b}$ de type pleuvoir, le schème syntaxique donné est A41: A = intransitif, $4: \mathrm{S}=$ complétive ou chose, 1 = locatif. Jamais on ne trouve la construction attendue A40: A41 rend compte des deux emplois de neiger dans le célèbre Il neige. Il neige sur Liège de Jacques Brel. Ceci oblige à considérer que le codage de construction reflète non pas l'usage, mais le sens, en l'occurrence l'image schématique, en tant que scène produite à l'interprétation : $L V F$ considère que les verbes météorologiques français impliquent toujours une représentation interprétative de la cible, qu'elle soit spécifiée ou non dans l'énoncé ; le schème syntaxique articule un couple trajecteur/ trajectoire (pleut) et une cible (à, sur, ou implicite). La construction A40, rejetée, correspond à un énoncé où le couple trajecteur/trajectoire pleut, uniquement associé à $i l$ impersonnel, ne serait dit de rien et ne ferait pas prédication. L'idée sous-jacente à cet encodage est que pour un verbe météorologique à incorporation nominale, le support de prédication ne peut être instancié par

\footnotetext{
${ }^{13}$ Pour une présentation de cette problématique, cf. Bottineau 1990 dans Langages 178, 91-95.

${ }^{14}$ Cf. l'analyse de me pudet «j'ai honte » (Lazard 1995, 283).
} 
il impersonnel seul pour cause de vacuité sémantique, aussi aura-t-on en complément une localisation, un support d'affect : l'évènement est dit du lieu qu'il concerne.

\begin{tabular}{|c|c|c|}
\hline brouillarder & ê.e.état brouillard brumasser & Il b sur la côte, dans la vallée. \\
\hline brouillasser & ê.e.état brouillard brumasser & Il $b \sim$ dans la plaine, sur la ville. \\
\hline bruiner & ê.e.état bruine faire de la bruine & Il b dans Paris, sur la côte. \\
\hline brumasser & ê.e.état brume crachiner & Il b sur la ville, sur la côte. \\
\hline brumer ê.e. & état brume brumasser & Il b sur la côte, dans la vallée. \\
\hline cailler 03 & ê.e.état froid geler & Il c ce matin. Ca $c \sim$ dans le bureau ce matin. \\
\hline crachiner & ê.e.état crachin brumasser & Il c sur Paris, sur la côte. \\
\hline dégeler 01 & ê.e.état dégel cesser de geler & Il d sur les routes. \\
\hline déglacer 02 & ê.e.état dégel dégeler, cesser de geler & Il d dans le nord du pays. \\
\hline dracher & ê.e.état pluie pleuvoir & Il d sur la côte. \\
\hline éclairer 09 & ê.e.état éclairs faire des éclairs & Il é dans le ciel. \\
\hline faire 15 & ê.e.état tps état de l'atmosphère & Il $f \sim$ de la pluie, de la neige, du vent. \\
\hline floconner 02 & ê.e.état flocons tomber de la neige & Il $f \sim$ sur la ville. Il $f \sim$ de gros flocons. \\
\hline flotter 01 & ê.e.état pluie pleuvoir & Il $f \sim$ sur la ville. \\
\hline frimasser & ê.e.état frimas $\mathrm{f}$ brouillard glaçant & Il $f \sim$ sur la ville. \\
\hline geler 08 & ê.e.état gel il glace & Il g sur la moitié nord du pays. \\
\hline givrer 05 & ê.e.état givre faire du givre sur & Il g sur la route, sur la voiture. \\
\hline glacer 07 & ê.e.état glace il fait très froid & Il g dans le nord. -ion \\
\hline grêlasser & ê.e.état grêle tomber un peu de grêle & Il g sur la ville. \\
\hline grêler 01 & ê.e.état grêle tomber de la grêle & Il g sur les champs. Il g de gros grêlons. \\
\hline grésiller 01 & ê.e.état grésil tomber du grésil & Il g $\sim$ sur la route. \\
\hline grisailler 01 & ê.e.état grisaille devenir gris, couvert & Il g sur la ville. \\
\hline lancequiner 01 & ê.e.état pluie pleuvoir & Il l sur la ville. \\
\hline lansquiner 01 & ê.e.état pluie pleuvoir & Il l sur la ville. \\
\hline mouillasser & ê.e.état crachin crachiner & Il m dans le jardin. \\
\hline neigeasser & ê.e.état neige peu neigeoter & Il n sur Paris. \\
\hline neigeoter & ê.e.état neige peu neigeasser & Il $n \sim$ sur Paris. \\
\hline neiger & ê.e.état neige tomber de la neige & Il $n \sim$ sur Paris. Il n de gros flocons. \\
\hline pincer 08 & ê.e.état froid geler & Ca p ce matin. \\
\hline pleuvasser & ê.e.état pluie peu tomber en pluie fine & Il p sur Paris. \\
\hline pleuviner & ê.e.état pluie peu tomber en petite pluie & Il p sur Paris. \\
\hline pleuvioter & ê.e.état pluie peu pleuvocher, pleuvoter & Il p sur Paris. \\
\hline pleuvocher & ê.e.état pluie peu pleuvioter, pleuvoter & Il p sur Paris. \\
\hline pleuvoir 01 & ê.e.état pluie tomber de la pluie & Il p sur Paris. Il p de grosses gouttes. \\
\hline pleuvoter & ê.e.état pluie peu pleuvocher, pleuvioter & Il p sur Paris. \\
\hline pluviner & ê.e.état pluie peu tomber de la pluie(f) & Il p sur Paris. \\
\hline reflotter 02 & ê.e.état+re pluie repleuvoir & Il $r \sim$ sur Paris. \\
\hline regeler 01 & ê.e.état+re gel faire du gel de nouveau & Il $r \sim$ ce matin à Paris. \\
\hline reglacer 01 & ê.e.état+re glace faire de la glace $\mathrm{d} n v$ & Il $r \sim$ ce matin à Paris. \\
\hline regrêler & ê.e.état+re grêle tomber grêle de nouveau & Il $r \sim$ sur le champ. \\
\hline reneiger & ê.e.état+re neige tomber neige de nouveau & Il $r \sim$ sur Paris. Il $r \sim$ de gros flocons. \\
\hline repleuvoir 01 & ê.e.état+re pluie tomber de la pluie & Il $r \sim$ sur Paris. Il $r \sim$ de grosses gouttes. \\
\hline repleuvoter & ê.e.état+re pluie tomber petite pluie & Il r sur Paris. \\
\hline retomber 02 & ê.e.état pluie, neige tomber une nvlle fois & La pluie, la neige, la grêle r \\
\hline reventer & ê.e.état+re vent venter de nouveau & Il r sur la côte. \\
\hline rosoyer & ê.e.état rosée tomber de la rosée & Il $r \sim$ sur le jardin. \\
\hline souffler 04 & ê.e.état vent venter & Ca $s \sim$ sur la plage. Le vent $s \sim$ fort. \\
\hline tomber 04 & ê.e.état pluie, neige descendre vers le sol & Il $t \sim$ de la neige.La neige $t \sim$. \\
\hline tonner 01 & ê.e.état tonnerre faire grondement sourd & Il $t \sim$ dans le lointain. Le ciel $t \sim$ au loin. \\
\hline vaser & ê.e.état pluie pleuvoir & Il v sur Paris ce matin. \\
\hline er 01 & ê.e.état vent le vent souffle & Il v fort sur la côte. \\
\hline verglacer 01 & ê.e.état verglas il se forme du verglas & Il v sur la route. \\
\hline
\end{tabular}

Par contraste, les verbes météorologiques de type fraîchir (abeausir, affraîchir, se raffraîchir) sont codés $\mathrm{A} 30: \mathrm{S}=$ chose, 0 = pas de circonstant ; mais leur sujet (le temps, le vent, la brise) 
n'est jamais $i l$ impersonnel seul (éventuellement ça déictique dans ça commence à fraîchir / se rafraîchir, mais pas $i l$ ) : le prédicat de transformation affecte un sujet non incorporé, et la condition implicite de prédication est satisfaite, à savoir, le fait que l'apport verbal soit dit d'une entité sémantiquement consistante. Au bout du compte, cela tendrait à prouver que il impersonnel français est réellement abstrait (au sens de: ne réfère pas à une entité interprétable), qu'il n'exprime pas la «personne d'univers» dans sa dimension métaphoriquement concrète (contrairement à l'ergatif impersonnel basque), qu'il peut structuralement être pris pour support de prédication (jouer le rôle d'amorçage du verbe, obligatoire en français et rendu nécessaire par l'incorporation nominale) mais pas de manière suffisante à lui seul faute de matière notionnelle, d'où le complément sémantique locatif explicite ou implicite postulé par l'encodage. Sur ce point $i l$ s'opposerait à ça, concret par son caractère déictique ; et au-delà du choix théorique opéré dans $L V F$, la distribution $* i l / c ̧ a / l e$ temps / le vent devant les verbes non incorporants de type fraîchir semble bien donner raison à cette analyse, pour contre-empirique et contre-intuitive qu'elle puisse paraitre au premier abord.

Signalons enfin que $L V F$ traite de manière unifiée les verbes météorologiques hyperonymiques comme pleuvoir et les verbes hyponymiques comme pleuviner, pleuvocher, pleuvasser. Or un rapide survol des faits d'usage observables dans les bases de données comme en français spontané capturé par moteur de recherche fait apparaître un système de corrélations que le dictionnaire ne peut pas intégrer. Ces verbes hyperonymiques sont utilisés pour spécifier la manière dont l'expérience du phénomène est vécue dans sa dimension objective (quantité, régularité) et subjective (image sous-jacente d'un agent pour crachiner) : ils dénotent autant la réalité du phénomène que la teneur du regard de l'observateur qui en fait l'expérience, autrement dit la relation phénoménologique. En raison de cet ancrage perceptuel et subjectif, ces verbes hyponymiques s'utilisent habituellement pour la description du phénomène par le locuteur qui l'a lui-même vécu ou en produit la mise en scène, si bien que le locus habituel est le « ici » de l'expérience, souvent pointé par le déictique : [à propos d'un lit-clos breton] On aimerait y rester quand ça crachine; ça crachine depuis ce matin. La localisation n'est pas exclue si elle est compatible avec ces nuances : il crachine de la neige sur le 95. Ces verbes sont totalement exclus de la phraséologie officielle des bulletins météorologiques en raison même de l'anonymat du présentateur qui se désincarne en tant que «non-expérienceur» du phénomène évoqué. Ils ne se prêtent pas davantage aux figements conventionnels, mais admettent les constructions par lesquels le locuteur manifeste la singularité de son regard par un emploi proche de la métaphore.

\section{Conclusion}

$L V F$ ne propose pas explicitement de traitement unitaire et intégrant de la question de l'impersonnel, et tel n'est pas son objectif d'ailleurs, s'agissant d'un dictionnaire. A cela, quatre raisons au moins :

(i) la coexistence d'un impersonnel sémantique et d'un impersonnel structural (verbes météorologiques vs autres cas) ;

(ii) la coexistence d'un impersonnel valenciel lexical et d'un impersonnel propositionnel syntaxique (falloir et verbes météorologiques vs arriver, rester etc ;

(iii) l'ambivalence du statut syntaxique de l'expansion (complétif, sujet réel, objet) ;

(iv) la coordination d'effets sémantiques et pragmatiques de natures distinctes sur les deux oppositions qui précèdent (et se chevauchent sans se superposer): la rhématisation et la mise en scène interlocutive pseudo-dialogale, commune à il pleut (quoi ?) des cordes, il est arrivé (quoi ?) un accident ; il faut (quoi ?) partir / 
qu'il parte; la quantification, commune à il part plus de soixante charrettes et $i l$ tombe des hallebardes.

Dans tous ces exemples, il impersonnel, en saturant le sujet par un marqueur aréférentiel, bloque l'instantiation du thème : il signale ou simule l'absence de consensus interlocutif quant à la nature du support de la prédication, le terme de départ destiné à faire l'objet d'un commentaire, une information, une transformation, une forme quelconque de mutation cognitive à l'interprétation. Par ce blocage, il impersonnel traduit un refus de fournir un repère informationnel initial : il suscite une mise en question et crée l'attente d'une réponse. Celle-ci est fournie par le «sujet réel », lequel précise le procès visé par la modalité (il faut partir), la quantification de l'agent (soixante charrettes), la nature du sujet inattendu ou non prototypique (il est arrivé un accident), les deux choses à la fois (il tombe des cordes).

Dans le cas du subjonctif, la mise en scène pseudo-dialogale se redouble d'un second degré à caractère polyphonique: à la question «lui, venir?» (la proposée de discours des guillaumiens), attribuée à un énonciateur structural sous-jacent, fictif mais identifiable à un pseudo-locuteur antérieur dans le dialogue, le locuteur refuse de répondre en formulant le jugement épistémique il se peut que (une «Attitude» dans le modèle de Ducrot), d'où la forme non-assertive du verbe (il vienne), interceptive et virtuelle dans le modèle guillaumien ( «saisie interceptive précoce et virtualisatrice » sur l'axe chronogénétique du temps opératif selon la psychomécanique). En syntaxe linéaire résultative, qu'il vienne répond à la question soulevée par $i l$ : l'expansion explicite la cible du jugement modal; et, selon une vision guillaumienne, en syntaxe génétique, au niveau de la transformée de discours, le subjonctif permet au locuteur de ne pas répondre à la question de l'actualisation soulevée par la proposée de discours. Ce n'est pas que nous tenions expressément à cette analyse psychomécanique, mais son explicitation permet de circonscrire précisément la nature de la mise en scène pseudo-dialogale due à il en vis-à-vis de la mise en scène polyphonique liée au mode subjonctif.

Le traitement de l'impersonnel dans LVF apparaît sous-tendu par ces principes :

(i) les entrées lexicalement impersonnelles de tel ou tel verbe ne sont signalées que lorsque l'usage crée un effet de coalescence entre l'impersonnel syntaxique au niveau de l'énoncé et les verbes particuliers qui, par une instanciation fréquente, en viennent à acquérir une valeur sémantique singularisable par figement et sédimentation (entrenchment en grammaire cognitive, traduit par «renforcement» en français, bien que le terme entrenchment véhicule bien plus cette notion de sédimentation);

(ii) le traitement des verbes météorologiques laisse transparaître une conception de la prédication selon laquelle il faut absolument qu'un support et un apport de signification sémantiquement consistants soient discernés à l'interprétation (et modélisables par exemples en termes d'images schématiques en linguistique cognitive), quitte à recourir à un circonstant locatif, explicite ou implicite, mais structuralement nécessaire, lorsqu'un sujet impersonnel ne fait pas l'affaire sémantiquement.

Par ces deux faits au moins, le traitement de l'impersonnel par LVF est symptomatique d'une théorisation sous-jacente, et elle n'est pas des moindres.

\section{Bibliographie}

Arrive M., GADET F., GALMiche M. (1986), La grammaire d'aujourd'hui. Paris : Flammarion

BALLY Ch. (193), Linguistique générale et linguistique française. Berne : Francke. 
BenVENiSTE E. ([1946] 1966), «Structure des relations de personne dans le verbe », in Problèmes de linguistique générale (1). Paris: Gallimard, 225-257.

Benveniste E. ([1950] 1966), «Actif et moyen dans le verbe », in Problèmes de linguistique générale (1). Paris: Gallimard, 168-175.

BERRENDONNER A. (1988), «Variations sur l'infinitif » in S. Rémi-Giraud (ed.), L'infinitif, Lyon: Presses Universitaires de Lyon, 149-165.

BERRENDONNER A. (2000), "Que reste-t-il de nos actants? Les passifs impersonnels en français », in P. Sériot \& A. Berrendonner (éds.), 43-53.

Boone Annie et Joly André, 1996, Dictionnaire terminologique de la systématique du langage, Paris, L'Harmattan.

Bottineau D. (2005), « Prédication et interaction cognitive en basque », i J. François \& I. Behr, Les constituants prédicatifs et la diversité des langues, Mémoires de la Société de Linguistique, XIV, Louvain : Peeters, 97-132.

Bottineau D. (2010a), «Typologie de la déflexivité », D. Bottineau, L. Begioni (éds.), La déflexivité, Langages 2010/2 (178), 89-113.

BotTinEAU D. (2010b), « La syntaxe phrastique actualise-t-elle l'incidence lexicale ? Etude comparée du français et du basque », M. Arabyan, J. Bres, D. Van Raemdonck, Th. Ponchon, R. Tremblay, P. Vachon-L'Heureux (éds.), Le Concept d'actualisation en psychomécanique, actes du XIIe Colloque international de l'Association internationale de psychomécanique du langage (AIPL), Bruxelles, 18-20 juin 2009, Limoges, LambertLucas, $\mathrm{xxx}-\mathrm{xxx}$. (sous presse)

BotTineau D. (2011), «Language and enaction », J. Stewart, O. Gapenne, E. Di Paolo (eds), Enaction: toward a new paradigm for cognitive science, MIT, 267-306.

Bottineau D. \& Roulland, D. (2010), «Les constructions impersonnelles en basque », F. Daviet-Taylor, D. Bottineau (dir.), L'impersonnel. Rennes: Presses Universitaires de Rennes, 243-263.

BRUNOT F. (1922), La pensée et la langue. Méthode, principes et plan d'une théorie nouvelle du langage appliquée au français. Paris : Masson, 1922.

Chocheyras, J. (1985), "Il me souvient" et "Je me souviens": de la cible à l'expérimentation », in J. Chocheyras et al., Autour de l'impersonnel. Grenoble : Ellug, 35-50.

CREISSELS D. (1991), «Approche des constructions asubjectales, improprement désignées comme “impersonnelles" », in M. Maillard (éd.), L'impersonnel. Mécanismes linguistiques et fonctionnements littéraires, Grenoble, CEDITEL, p. 47-57.

CReissels, D., Cours de syntaxe générale 2004, en ligne, Chapitre 11 Constructions impersonnelles.

CREISSELS D. (2006), Syntaxe générale, une introduction typologique, Paris : Hermès.

Charaudeau P. (1992), Grammaire du sens et de l'expression. Paris : Hachette.

DAVIET-TAYlor F., BottineAu D. (2010), L'impersonnel: le verbe, la personne, la voix. Rennes : Presses Universitaires de Rennes.

De CaRvalho P. (1991), «L'impersonnel, un «effet de sens »? Sur les «impersonnels météorologiques », du latin, in M. Maillard (dir.), L’Impersonnel, Mécanismes linguistiques et fonctionnements littéraires, Actes du Colloque de Grenoble (1990), CEDITEL, Grenoble.

De CARvalho P. (2010), «La fin d'une illusion, ou l'impersonnel réduit et ramené à moi », in F. Daviet-Taylor, D. Bottineau (éds), L'impersonnel: le verbe, la personne, la voix. Rennes : Presses Universitaires de Rennes, 53-71.

DouAY C. (2000), Éléments pour une théorie de l'interlocution. Rennes: Presses Universitaires de Rennes.

Dubois J. \& Dubois-ChARLIER F. (1997), Les Verbes français. Paris : Larousse-Bordas. 
FeUILLET J. (1998), Actance et valence dans les langues d'Europe, Mouton de Gruyter, Berlin $\&$ New York.

FEUILLET J. (2006), Introduction à la typologie linguistique, Paris : Champion.

GAATONE D. (2000), «Les phrases à sujet postiche », in Mélanges de linguistique, sémiotique et narratologie dédiés à la mémoire de Krassimir Mantchev. Sofia: Colibri.

Grevisse M. - A. Goosse, 1993, Le bon usage. Grammaire française, $13^{\mathrm{e}}$ édition, Paris Louvain-la-Neuve : Duculot.

Goldberg A. E. (1995), Constructions: A Construction Grammar Approach to Argument Structure, University of Chicgo Press.

Guillaume G. (1970), Temps et verbe : théorie des aspects, des modes et des temps, Paris : Champion.

Guillaume G. (1971-1999), Leçons de linguistique, 17 vol., Québec: Les Presses de l'Université Laval, Lille : Presses Universitaires de Lille.

Guillaume G. (1964), Langage et science du langage, Paris : Nizet, Québec: Presses de l'Université Laval.

HERIAU M. (1984), «Impersonnel et transitivité», Tétralogies $n^{\circ} 1$, Problèmes de glossologie, Rennes : Presses Universitaires de Rennes.

KeEnAN E. (1976), «Towards a Universal Definition of Subject» in L. Charles \& S. Thompson (eds.), Subjects and topics. New York: Academic Press, 305-333.

KEMMER S. (1993) The Middle Voice. Amsterdam /Philadelphia: Benjamins.

KESIK M. (1985), «La phrase impersonnelle : problèmes de description », in J. Chocheyras et al., Autour de l'impersonnel, Grenoble : Ellug, 51-62.

KLEIBER G. (1993), «Iconicité d'isomorphisme et grammaire cognitive », Faits de langue I, 105-121.

LAMBERT P.-Y. (1997), "L'impersonnel”, Actance et valence dans les langues d'Europe, J. Feuillet (ed)., Mouton de Gruyter.

LAVIE R.-J. (2010), « Pour une approche non spécifique du TAM (temps, aspect, modalité) » in C. Douay (éd.), Système et chronologie. Rennes : Presses Universitaires de Rennes, 4169.

LAZARD, G. (1994a), L'Actance. Paris : PUF.

LAZARD G. (1994b) : «L'actant H : sujet ou objet ? in Bulletin de la Société de Linguistique 89. Louvain : Peeters, 1-24.

LAZARD G. (1999), «Pour une terminologie rigoureuse : quelques principes et propositions », in Mémoires de la Société de Linguistique de Paris, La terminologie linguistique, Tome VI. Louvain : Peeters.

LAZARD G. (2008), « La prédication implique-t-elle un sujet? », Faits de Langues 31-32, 67 75.

LE Bidois G, LE Bidois R. (1935), Syntaxe du français moderne. Tome I. Paris : Auguste Picard.

LE BIDOIS G. \& LE BIDOIS R. (1938), Syntaxe du français moderne. Tome II. Paris : Auguste Picard.

LETOUBLON F. (1994), « La personne et ses masques », Faits de langues 3.

MAILlARD M. (1985), "L'impersonnel français de "il" à "ça" », in J. Chocheyras et al., Autour de l'impersonnel. Grenoble : Ellug, 63-118.

MAILlARD M. (dir.) (1991), L'impersonnel. Mécanismes linguistiques et fonctionnements littéraires. Grenoble : Ceditel \& Lidilem.

MAILLARD M. (éd.) (1994), L'impersonnel, L'Information grammaticale, 62.

MAILlARD M. (2008), «Y a-t-il prédication sans sujet ni verbe? » ? Faits de Langues 31-32, 23-32.

MAILlARD M. \& ALMEIDA E. (2000), «Un modèle nodal pour une description cohérente de l'impersonnel en français et en portugais », in P. Sériot, A. Berrendonner (éds.), Le 
paradoxe du sujet. Les propositions impersonnelles dans les langues slaves et romanes, Cahiers de l'ILSL, 12. Lausanne: UNIL.173-206.

Moignet G. (1970), «Personne humaine et personne d'univers : contributions à l'étude du verbe unipersonnel », Mélanges Albert Henri, Paris: Centre de Philologie et de Littératures romanes de l'Université de Strasbourg.

Moignet G. (1974), Etudes de Psycho-systématique française. Paris : Klincksieck.

Moignet G. (1981), Systématique de la langue française. Paris : Klincksieck.

Morel, M.-A. (2010), « Déflexivité et décondensation dans le dialogue oral enfrançais », D. Bottineau, L. Begioni (éds.), La déflexivité, Langages 2010/2 (178), 115-131.

Riegel M., Pellat J.-C. \& Rioul R. (1994), Grammaire méthodique du français. Paris : PUF.

RotaetXe, K. (1998), «L'actance en basque », in Feuillet, J. (éd.), Actance et valence dans les langues d'Europe, Mouton De Gruyter, Berlin / New York, 849-879.

Rousseau, André (1994): «L'énoncé impersonnel en allemand» in : L'Information grammaticale $62\left(\mathrm{n}^{\circ}\right.$ spécial dirigé par M. Maillard) pp. 23-28.

Rousseau, André (2010): "L'énoncé impersonnel en typologie des langues et en philosophie du langage », in F. Daviet-Taylor, D. Bottineau (éds), L'impersonnel: le verbe, la personne, la voix. Rennes : Presses Universitaires de Rennes, 31-52.

RUWET N. (1990), « Des expressions météorologiques », Le français moderne, 58, 43-97.

SANDFELD K. (1970), Syntaxe du français contemporain. I. Les pronoms. Paris : Champion.

SERIOT P. \& BERRENDONNER A. (éds.) (2000), Le paradoxe du sujet. Les propositions impersonnelles dans les langues slaves et romanes, Cahiers de l'ILSL, 12. Lausanne: UNIL.

TESNIERE L. (1959), Éléments de syntaxe structurale. Paris: Klincksieck.

TOGO Y. (1994), «Impersonnel, passif et potentiel: une approche contrastive du français et du japonais », in M. Maillard (éd.), L'impersonnel, L'Information grammaticale, 62, 39-41.

WAGNER R. L., PINCHON J. (1962), Grammaire du français classique et moderne. Paris : Hachette.

Wartburg W. VON, ZuMthor P. (1947), Précis de syntaxe du français contemporain. Berne : Francke. 